\title{
MANIFOLDS AND STIEFEL-WHITNEY CLASSES
}

\author{
MARK ANDERSON
}

(Communicated by Haynes R. Miller)

\begin{abstract}
This paper constructs manifolds which generate the unoriented cobordism ring having a maximum number of vanishing high dimensional Stiefel-Whitney classes.
\end{abstract}

\section{INTRODUCTION}

This paper studies the ring $\mathscr{N}_{*}$ of unoriented cobordism classes of closed smooth manifolds. Thom [9] has shown that $\mathscr{N}_{*}$ is isomorphic to $\mathbf{Z}_{2}\left[x_{i} \mid i \neq\right.$ $\left.2^{s}-1\right]$ the $\mathbf{Z}_{2}$ polynomial ring with generators $x_{i}$ for all natural numbers $i$ not of the form $2^{s}-1$. The most common generators to work with are those represented by $\mathbf{R} P^{2 n}$ in even dimensions and by certain Dold manifolds [2] in odd dimensions. The purpose of this paper is to provide a new set of generators whose nonzero Stiefel-Whitney numbers involve only low dimensional StiefelWhitney classes.

Stong and Yoshida [8] have shown that high dimensional nonbounding manifolds have high dimensional Wu classes. More precisely,

Proposition. For any integer $k$ there is an integer $N=N(k)$ so that a closed manifold $M$ with $W u$ class $v=1+v_{1}+\cdots+v_{k}$ bounds if $\operatorname{dim} M>N$.

It is noted that no similar result holds for Stiefel-Whitney classes. What is proven in this paper is the following.

Theorem. If $M^{n}$ is a closed smooth n-manifold with Stiefel-Whitney class given by $w=1+w_{1}+\cdots+w_{k}$, where $k$ is less than or equal to the number of ones in the dyadic expansion of $n$, then $M$ is decomposable in $\mathscr{N}_{*}$.

This paper then, constructs $n$-manifolds $M^{n}$ for each positive integer $n$ not of the form $2^{s}-1$ where each manifold $M^{n}$ represents an indecomposable in $\mathscr{N}_{*}$ and has Stiefel-Whitney class $w=1+w_{1}+\cdots+w_{k+1}$ where $k$ is the number of ones in the dyadic expansion of $n$.

Received by the editors July 1, 1987 and, in revised form, December 2, 1987.

1980 Mathematics Subject Classification (1985 Revision). Primary 57R20.

Key words and phrases. Indecomposable manifold, Stiefel-Whitney class. 
The author wishes to thank Robert E. Stong for his help in writing the dissertation from which this paper comes.

\section{THE MAIN THEOREM}

Let $M^{n}$ be an $n$-manifold. Let $f: M \rightarrow B O$ be the classifying map for the tangent bundle over $M, f^{*}: H^{*}(B O) \rightarrow H^{*}(M)$ the associated homomorphism in cohomology with $\mathbf{Z}_{2}$ coefficients (as are all coefficients in this paper), and $\phi: H^{*}(B O(m)) \rightarrow H^{*}\left(B \mathbf{Z}_{2}^{m}\right)$ the monomorphism induced by the inclusion $i: \mathbf{Z}_{2}^{m} \hookrightarrow O(m) . \quad H^{*}\left(B \mathbf{Z}_{2}^{m}\right)$ is isomorphic to $\mathbf{Z}_{2}\left[x_{1}, \ldots, x_{m}\right]$ the $\mathbf{Z}_{2}$ polynomial ring with $m$ one-dimensional generators, and the image of $\phi$ to the subring of symmetric polynomials. Recognizing that $H^{i}(B O) \rightarrow H^{i}(B O(m))$ is an isomorphism for $i \leq m$ one can make the following definitions. The total Stiefel-Whitney class $w$ of $M$ is given by

$$
w=f^{*} \circ \phi^{-1}\left[\prod_{i=1}^{m}\left(1+x_{i}\right)\right] \text {, }
$$

where $m$ is sufficiently large, and the $k$ th Stiefel-Whitney class $w_{k}$ by

$$
w_{k}=f^{*} \circ \phi^{-1}\left[\sum x_{1} x_{2} \cdots x_{k}\right]
$$

where $\sum x_{1} x_{2} \cdots x_{k}$ is the smallest symmetric polynomial in $\mathbf{Z}_{2}\left[x_{1}, \ldots, x_{m}\right]$ containing the term $x_{1} x_{2} \cdots x_{k}$. The total Wu class $v$ and the $k$ th $s$-class $s_{k}$ are then defined by

$$
v=\mathrm{Sq}^{-1} w=f^{*} \circ \phi^{-1}\left[\prod_{i=1}^{m}\left(1+x_{i}+x_{i}^{2}+x_{i}^{4}+\cdots\right)\right]
$$

and

$$
s_{k}=f^{*} \circ \phi^{-1}\left[\sum_{i=1}^{m} x_{i}^{k}\right] \text {. }
$$

Due to the fact that $s_{k}(B O(m))$ is a primitive element in $H^{*}(B O)$ for all $k$, the class of $M^{n}$ is indecomposable in $\mathscr{N}_{*}$ if and only if $s_{n}\left(\mu_{M}\right)=1\left(\mu_{M}\right.$ or $\mu$ will denote the fundamental homology class of $M)$. Also, if $k=2^{r}-1$ for some $r$, then cupping with $s_{k}$ is actually a cohomology operation into $H^{n}\left(M^{n}\right)$. This is seen as follows.

If $Q_{k} \in \mathscr{A}$ is defined inductively by $Q_{0}=\mathrm{Sq}^{1}$ and $Q_{k}=\mathrm{Sq}^{2^{k}} Q_{k-1}+$ $Q_{k-1} \mathrm{Sq}^{2^{k}}$, then $Q_{k}$ is a primitive element in $\mathscr{A}$ [3].

Proposition. $Q_{k}(x)=S_{2^{k}-1} \cup x$ for any $x \in H^{n-2^{k}+1}\left(M^{n}\right)$.

Proof. k Define $\psi: \mathscr{A} \rightarrow H^{*}(M O)$ by $\psi(\alpha)=\alpha(u)$ where $u$ is the Thom class in $H^{*}(B O) . \psi$ is a monomorphism and so $\psi\left(Q_{k}\right)=p \cup u$, where $p$ is a primitive in $H^{*}(B O)$ of dimension $2^{k}-1$. Hence, $\psi\left(Q_{k}\right)=S_{2^{k}-1} \cup u$ since the $s$-classes are the only primitives in $H^{*}(B O)$. Then, by Poincare duality, $Q_{k}(x)=s_{2^{k}-1} \cup x$ for any $x \in H^{n-2^{k}+1}\left(M^{n}\right)$. 
Let $\alpha(n)$ denote the number of ones in the dyadic expansion of $n$, i.e., $\alpha(n)=r$ if $n=2^{\alpha_{1}}+2^{\alpha_{2}}+\cdots+2^{\alpha_{r}}$ with $\alpha_{1}<\alpha_{2}<\cdots<\alpha_{r}$. We are ready to prove the main theorem.

Proof. Let $M^{n}$ be an $n$-manifold with $w=1+w_{1}+\cdots+w_{k}$ where $k=\alpha(n)$. Write $n=2^{\alpha_{1}}+2^{\alpha_{2}}+\cdots+2^{\alpha_{k}}$ where $0 \leq \alpha_{1}<\cdots<\alpha_{k}$. We need to show that $s_{n}\left(\mu_{M}\right)=0$.

Case 1. $n$ even. Let $q=1+2^{\alpha_{1}}+2^{\alpha_{2}}+\cdots+2^{\alpha_{k-1}}$. Then

$$
\begin{aligned}
s_{n} & =f^{*} \circ \phi^{-1}\left[\sum_{i=1}^{k}\left(x_{i}^{n}\right)\right]=\operatorname{Sq}^{q}\left[f^{*} \circ \phi^{-1}\left[\sum_{i=1}^{k}\left(x_{i}^{2^{\mu_{k}-1}}\right)\right]\right]=v_{q} \cup s_{\left(2^{\left.\alpha_{k}-1\right)}\right.} \\
& =s_{\left(2^{\left.n_{k}-1\right)}\right.}\left[f^{*} \circ \phi^{-1}\left(\sum x_{1} x_{2}^{2^{\alpha_{1}}} \cdots x_{r}^{2^{n_{k}-1}}\right)\right] .
\end{aligned}
$$

Since $s_{\left(2^{\prime \prime} k-1\right)}$ is a primitive operation,

$$
s_{\left(2^{\prime \prime k}-1\right)}\left(v_{q}\right)=f^{*} \circ \phi^{-1}\left[\sum x_{1}^{2^{\prime \prime k}} x_{2}^{2^{n_{1}}} \cdots x_{r}^{2^{n_{k}-1}}\right]=v_{n}=0 .
$$

So $s_{n}\left(\mu_{M}\right)=0$ and $M$ is decomposable in $\mathscr{N}_{*}$.

Case 2. $n$ odd. Let $q=n-2^{\alpha_{k}}+1=2^{a}+2^{\alpha_{a+1}}+\cdots+2^{\alpha_{k-1}}$ (so $\alpha_{i}+1=\alpha_{i+1}$ for $i<a$ and $\left.\alpha_{a}+1<\alpha_{a+1}\right)$. Then

$$
s_{n}=\mathrm{Sq}^{q}\left[f^{*} \circ \phi^{-1}\left[\sum_{i=1}^{k}\left(x_{i}^{2^{\prime \prime} k-1}\right)\right]\right]=v_{q} \cup s_{\left(2^{\prime \prime k}-1\right)}=s_{\left(2^{\prime \prime k}-1\right)}\left(v_{q}\right) \text {. }
$$

Since $s_{\left(2^{\prime \prime} k-1\right)}$ is a primitive operation, $s_{\left(2^{\prime \prime} k-1\right)}\left(y^{2}\right)=0$ for any $y^{2} \in H^{q}(M)$. So we need only write $v_{q}$ modulo terms which are squares. Hence,

$$
\begin{aligned}
s_{\left(2^{\prime \prime k}-1\right)}\left(v_{q}\right) & =s_{\left(2^{\left.a_{k}-1\right)}\right.}\left[f^{*} \circ \phi^{-1}\left(\sum x_{1} x_{2} x_{3}^{2} x_{4}^{4} \cdots x_{a+1}^{2^{a-1}} x_{a+2}^{2^{\prime \prime} a+1} \cdots x_{r}^{2^{2^{\prime \prime}-1}}\right)\right] \\
& =f^{*} \circ \phi^{-1}\left[\sum x_{1}^{2^{a_{k}}} x_{2} x_{3}^{2} \cdots x_{r}^{2^{\prime \prime k-1}}\right]=v_{n}=0 .
\end{aligned}
$$

Hence, $s_{n}\left(\mu_{M}\right)=0 \in \mathbf{Z}_{2}$. Thus, $M$ is decomposable and we have shown that every indecomposable $n$-manifold $M^{n}$ has a nonzero $i$ th Stiefel-Whitney class $w_{i}$ with $i>\alpha(n)$.

\section{Constructions}

For each positive integer $n$ not of the form $2^{s}-1$, we construct an indecomposable $n$-manifold with $w_{k}=0$ for all $k>\alpha(n)+1$.

We will denote by $\mathbf{R} P\left(m_{1}, m_{2}, \ldots, m_{k}\right)$ the manifold fibered over $\mathbf{R} P^{m_{1}} \times$ $\mathbf{R} P^{m_{2}} \times \cdots \times \mathbf{R} P^{m_{k}}$ with fiber $\mathbf{R} P^{k-1}$ :

$$
\mathbf{R} P\left(m_{1}, \ldots, m_{k}\right)=\mathbf{R} P\left(\begin{array}{c}
\lambda_{1} \oplus \cdots \oplus \lambda_{k} \\
\bigsqcup_{\mathbf{R} P^{m_{1}} \times \cdots \times \mathbf{R} P^{m_{k}}}
\end{array}\right)
$$


where $\lambda_{i}$ is the pullback of the standard line bundle over $\mathbf{R} P^{m_{i}}$. Notice

$$
\mathbf{R} P\left(m_{1}, \ldots, m_{k}\right)
$$

has dimension $n=\left[\sum_{i=1}^{k}\left(m_{i}+1\right)\right]-1$. These manifolds are nice to work with because both the Stiefel-Whitney class $w$ and the $s$-number $s_{n}\left(\mu_{M^{n}}\right)$ are easy to compute. If $M$ is $\mathbf{R} P\left(m_{1}, \ldots, m_{k}\right), c$ the first Stiefel-Whitney class of the standard line bundle over $M, \sum_{i_{1}<\cdots<i_{j}} a_{i_{1}} a_{i_{2}} \cdots a_{i_{j}}$ the $j$ th StiefelWhitney class of $\lambda_{1} \oplus \cdots \oplus \lambda_{k}$ where $a_{t}$ is the pullback of the nonzero class in $H^{1}\left(\mathbf{R} P\left(m_{t}\right)\right)$, then $w(M)=\prod_{i=1}^{k}\left(1+a_{i}\right)^{m_{i}+1}\left(1+a_{i}+c\right)$ with the relations $a_{i}^{m_{i}+1}=0$ and $c^{k}=\left(\sum_{i=1}^{k} a_{i}\right) c^{k-1}+\left(\sum_{i<j} a_{i} a_{j}\right) c^{k-2}+\cdots+a_{1} a_{2} \cdots a_{k}$. And

$$
s_{n}\left(\mu_{M}\right)=\sum_{i=1}^{k}\left(\begin{array}{c}
n-1 \\
m_{i}
\end{array}\right) \bmod 2 .
$$

Hence, $M$ is indecomposable if and only if $\sum_{i=1}^{k}\left(\begin{array}{c}n-1 \\ m_{i}\end{array}\right)$ is odd [7]. Now we are prepared to give the promised manifolds. First we will tackle the case of even dimensions.

Even case. If $n$ is even, then $n$ can be written uniquely as $n=2^{\alpha_{t}}+2^{\alpha_{t-1}}+$ $\cdots+2^{\alpha_{1}}+2^{\alpha_{0}}$, where $\alpha_{t}>\alpha_{t-1}>\cdots>\alpha_{1}>\alpha_{0}>0$.

$$
M=\mathbf{R} P\left(2^{\alpha_{t}}-1,2^{\alpha_{t-1}}-1, \ldots, 2^{\alpha_{1}}-1,2^{\alpha_{0}-1}-1,2^{\alpha_{0}-1}-1,0\right)
$$

is an indecomposable $n$-manifold with $w_{k}=0$ for all $k>\alpha(n)+1$. First we check the dimension of $M . \operatorname{Dim}(M)=\sum_{i=1}^{t}\left(2^{\alpha_{i}}-1+1\right)+2\left(2^{\alpha_{0}-1}-1+1\right)+$ $1-1=n$. Second, to see that $M$ is indecomposable, we show that

$$
s=\sum_{i=1}^{t}\left(\begin{array}{c}
n-1 \\
2^{\alpha_{i}}-1
\end{array}\right)+2\left(\begin{array}{c}
n-1 \\
2^{\alpha_{0}-1}-1
\end{array}\right)+\left(\begin{array}{c}
n-1 \\
0
\end{array}\right)
$$

is odd. For $i \geq 1$ (i.e., $2^{\alpha_{i}} \geq 4$ ),

$$
\left(\begin{array}{c}
n-1 \\
2^{\alpha_{i}}-1
\end{array}\right)=\left(\begin{array}{c}
2^{\alpha_{i}}+\cdots+2^{\alpha_{1}}+2^{\alpha_{0}-1}+2^{\alpha_{0}-2}+\cdots+2+1 \\
2^{\alpha_{i}-1}+\cdots+2^{\alpha_{0}}+\cdots+1
\end{array}\right)
$$

is even. Here we are using a lemma proven in Steenrod [6].

Lemma. Let $a=\sum_{i=0}^{m} a_{i} 2^{i}$ and $b=\sum_{i=0}^{m} b_{i} 2^{i} \quad\left(0 \leq a_{i}, b_{i} \leq 1\right)$. Then

$$
\left(\begin{array}{l}
b \\
a
\end{array}\right)=\prod_{i=0}^{m}\left(\begin{array}{l}
b_{i} \\
a_{i}
\end{array}\right) \bmod 2
$$

Since the next term is also even and $\left(\begin{array}{c}n-1 \\ 0\end{array}\right)$ odd, $s$ is odd and $M$ indecomposable.

Third, we compute the Stiefel-Whitney class of $M . w=\prod_{i=1}^{t+3}\left(1+a_{i}+c\right)$ where $a_{t+3}=0$ and $c^{t+3}=\left(\sum a_{i}\right) c^{t+2}+\cdots+\left(a_{1} a_{2} \cdots a_{t+2}\right) c$. Hence, $w_{t+3}=$ $c^{t+3}+\left(\sum a_{i}\right) c^{t+2}+\cdots+\left(a_{1} \cdots a_{t+2}\right) c=0$. So $w_{k}=0$ if $k>t+2=\alpha(n)+1$ and we have finished the case of even dimensions.

Odd case. In order to construct the desired manifolds with odd dimension, we need to use a fact proven by Stong [7]. 
Proposition. If an indecomposable $(m+p)$-manifold $M^{\prime}$ fibers over an $m$ manifold $B^{\prime}$ and $f: B \rightarrow B^{\prime}$ is a degree-one map from an m-manifold $B$ into $B^{\prime}$, then the induced fibration $M^{m+p}=f^{*}\left(M^{\prime}\right)$ over $B$ is an indecomposable manifold.

We now move on to construct odd dimensional $n$-manifolds with $w=1+$ $w_{1}+\cdots+w_{k}$ where $k=\alpha(n)+1$. Given $n$ odd, write $n=2^{\alpha_{t}}+2^{\alpha_{t-1}}+\cdots+$ $2^{\alpha_{1}}+2^{\alpha_{0}}$, where $\alpha_{t}>\cdots>\alpha_{1}>\alpha_{0}=0$. Let $q=\min \left\{i \mid i \neq \alpha_{i}\right\}$, that is

$$
\alpha_{q}=\min \left\{\alpha_{i} \mid \alpha_{i}>\alpha_{i-1}+1\right\} \text {. }
$$

As a first attempt, set

$$
M^{\prime}=\mathbf{R} P\left(2^{\alpha_{t}}-1, \ldots, 2^{\alpha_{q+1}}-1,2^{\alpha_{q}-1}, 2^{\alpha_{q}-1}-1,2^{\alpha_{q-1}}-1, \ldots, 2^{\alpha_{0}}-1\right) .
$$

As before, we need to check the dimension of $M^{\prime}$, that $M^{\prime}$ is indecomposable and that the top nonzero Stiefel-Whitney class of $M^{\prime}$ has low dimension. First, $M^{\prime}$ has dimension $\operatorname{dim}(M)=\sum_{i=0, i \neq q}^{t}\left(2^{\alpha_{i}}\right)+\left(2^{\alpha_{q}-1}+1\right)+\left(2^{\alpha_{q}-1}\right)-1=n$. Second, we calculate

$$
s=\sum_{\substack{i=0 \\
i \neq q}}^{t}\left(\begin{array}{c}
n-1 \\
2^{\alpha_{i}}-1
\end{array}\right)+\left(\begin{array}{c}
n-1 \\
2^{\alpha_{q}-1}
\end{array}\right)+\left(\begin{array}{c}
n-1 \\
2^{\alpha_{q}-1}-1
\end{array}\right)+\left(\begin{array}{c}
n-1 \\
2^{\alpha_{0}}-1
\end{array}\right) .
$$

Since $\left(\begin{array}{c}2 j \\ 2 k+1\end{array}\right)$ is even for any nonnegative integers, we only need to consider the terms

$$
\left(\begin{array}{c}
n-1 \\
2^{\alpha_{q}-1}
\end{array}\right) \text { and }\left(\begin{array}{c}
n-1 \\
2^{\alpha_{0}}-1
\end{array}\right) .
$$

The first,

$$
\left(\begin{array}{c}
n-1 \\
2^{\alpha_{q}-1}
\end{array}\right)=\left(\begin{array}{c}
2^{\alpha_{t}}+\cdots+2^{\alpha_{q}}+2^{\alpha_{q-1}}+\cdots+2^{\alpha_{1}} \\
2^{\alpha_{q}-1}
\end{array}\right)
$$

is even since $\alpha_{q}>\alpha_{q}-1>\alpha_{q-1}$. The second is odd since $2^{\alpha_{0}}-1=0$ and $\left(\begin{array}{c}n-1 \\ 0\end{array}\right)=1$. Thus $s$ is odd and $M^{\prime}$ is indecomposable. Unfortunately, the Stiefel-Whitney class of $M^{\prime}$ is not quite what we want. Because $w$ has a factor $\left(1+a_{q}\right)^{2^{n_{4}-1}+1}=\left(1+a_{q}+a_{q}^{2^{n+}-1}\right)$ coming from $H^{*}\left(\mathbf{R} P^{2^{n_{q}}-1}\right)$, it has high dimensional terms. It is true that every Stiefel-Whitney number divisible by $w_{k}$ with $k>\alpha(n)+1$ is zero, but by using the proposition proven at the start of this case, we can get $w_{k}$ actually equal to zero for all $k>\alpha(n)+1$. Let $B^{\prime}$ be the base space of $M^{\prime}$ and $B$ the manifold fibered over

$$
\begin{aligned}
\mathbf{R} P^{2^{\alpha_{t}}-1} \times \cdots & \times \mathbf{R} P^{2^{\prime \prime q} q+1} \times \mathbf{R} P^{2^{a_{q}-1}-1} \\
& \times \mathbf{R} P^{2^{a_{q}-1}-1} \times \mathbf{R} P^{2^{n_{q} q-1}-1} \times \cdots \times \mathbf{R} P^{0}
\end{aligned}
$$

with fiber $\mathbf{R} P(\lambda \oplus 1)$ where $\lambda \oplus 1$ is the pullback of the standard line bundle plus a trivial line bundle over the first $\mathbf{R} P^{2^{\prime \prime 4}-1}-1$ factor. Then, recognizing that the 
bundle is isomorphic to the connected sum $\mathbf{R} P^{n} \sharp \mathbf{R} P^{n}$ [7], one lets $f: B \rightarrow B^{\prime}$ be the map induced by

$$
f: \downarrow_{\mathbf{R} P^{2^{\prime \prime}-1}-1} \stackrel{\mathbf{R} P(\lambda \oplus 1)}{\cong} \mathbf{R} P^{2^{\prime \prime}-1} \sharp \mathbf{R} P^{2^{\prime \prime}-1} \rightarrow \mathbf{R} P^{2^{\prime \prime q}-1},
$$

the map which collapses one summand to a point. Then $f$ is a degree-one map and the pullback $M=f^{*}\left(M^{\prime}\right)$ is an indecomposable manifold fibered over

$$
\begin{aligned}
\mathbf{R} P^{2^{\prime \prime t}-1} \times \cdots & \times \mathbf{R} P^{2^{\prime \prime q} q+1} \times \mathbf{R} P^{2^{\alpha_{q}-1}-1} \\
& \times \mathbf{R} P^{2^{\alpha_{q}-1}-1} \times \mathbf{R} P^{2^{\alpha^{\prime \prime} q-1}-1} \times \cdots \times \mathbf{R} P^{0} .
\end{aligned}
$$

Since the tangent bundle over $\mathbf{R} P^{2^{\prime \prime} i-1}$ has $w=1$, for all $i$, one has that $w_{k}=0$ if

$$
k \geq n-\left[\sum_{\substack{i=0 \\ i \neq q}}^{t}\left(2^{\alpha_{i}}-1\right)+2\left(2^{\alpha_{4}-1}-1\right)\right]=n-(n-t-3)=t+3=\alpha(n)+2,
$$

that is, $w=1+w_{1}+\cdots+w_{k}$ with $k=\alpha(n)+1$. We are then finished with constructions.

\section{AFTERWORD}

One would like to replace "is decomposable" with "bounds" in this theorem. However, one has then set about to solve a much harder problem. One would need to show all Stiefel-Whitney numbers vanish rather than just the $s$-number $s_{n}(\mu)$. It is however, conjectured that every $n$-manifold bounds if $w_{k}=0$ for all $k>\alpha(n)$.

\section{REFERENCES}

1. P. E. Conner and E. E. Floyd, Differentiable periodic maps, Springer-Verlag, Berlin, 1964.

2. A. Dold, Erzeugende der Thomschen Algebra $\mathscr{N}$, Math. Z. 65 (1956), 25-35.

3. J. W. Milnor, The Steenrod algebra and its dual, Ann. of Math. (2) 67 (1958), 150-171.

4. J. W. Milnor and J. D. Stasheff, Characteristic classes, Princeton Univ. Press, Princeton, N. J., 1974.

5. L. S. Pontrjagin, Characteristic cycles on differentiable manifolds, Math. Sb. (N.S.) 21 (1947), 233-284; Amer. Math. Soc. Transl. 32 (1950).

6. N. E. Steenrod, Cohomology operations, Princeton Univ. Press, Princeton, N. J., 1962.

7. R. E. Stong, On fiberings of cobordism classes, Trans. Amer. Math. Soc. 178 (1973), 431-447.

8. R. E. Stong and T. Yoshida, Wu classes, Proc. Amer. Math. Soc. 100 (1987), 352-354.

9. R. Thom, Quelques propriétés globales des variétés differentiables, Comment. Math. Helv. 28 (1954), 17-86.

10. C. T. C. Wall, Determination of the cobordism ring, Ann. of Math. (2) 72 (1960), 292-311. 